\title{
The genome sequence of the bramble shoot moth, Notocelia
}

\section{uddmanniana (Linnaeus, 1758) [version 1; peer review:}

\section{awaiting peer review]}

\section{Douglas Boyes ${ }^{1+}$, Peter W.H. Holland (1D), Darwin Tree of Life Barcoding collective, Wellcome Sanger Institute Tree of Life programme, \\ ${ }^{1}$ UK Centre for Ecology and Hydrology, Wallingford, Oxfordshire, UK \\ ${ }^{2}$ Department of Zoology, University of Oxford, Oxford, UK \\ + Deceased author} University of Oxford and Wytham Woods Genome Acquisition Lab, Wellcome Sanger Institute Scientific Operations: DNA Pipelines collective, Tree of Life Core Informatics collective, Darwin Tree of Life Consortium

\section{V1 First published: $15 \operatorname{Dec} 2021, \mathbf{6 : 3 4 8}$}

https://doi.org/10.12688/wellcomeopenres.17488.1

Latest published: 15 Dec 2021, 6:348

https://doi.org/10.12688/wellcomeopenres.17488.1

\section{Abstract}

We present a genome assembly from an individual male Notocelia uddmanniana (the bramble shoot moth; Arthropoda; Insecta; Lepidoptera; Tortricidae). The genome sequence is 794 megabases in span. The majority of the assembly, $99.96 \%$, is scaffolded into 28 chromosomal pseudomolecules, with the $Z$ sex chromosome assembled.

\section{Keywords}

Notocelia uddmanniana, bramble shoot moth, genome sequence, chromosomal, Lepidoptera

\section{Open Peer Review}

Approval Status AWAITING PEER REVIEW

Any reports and responses or comments on the article can be found at the end of the article.

This article is included in the Tree of Life

gateway. 
Corresponding author: Darwin Tree of Life Consortium (mark.blaxter@sanger.ac.uk)

Author roles: Boyes D: Investigation, Resources; Holland PWH: Supervision, Writing - Original Draft Preparation, Writing - Review \& Editing;

Competing interests: No competing interests were disclosed.

Grant information: This work was supported by Wellcome through core funding to the Wellcome Sanger Institute (206194) and the Darwin Tree of Life Discretionary Award (218328).

The funders had no role in study design, data collection and analysis, decision to publish, or preparation of the manuscript.

Copyright: (c) 2021 Boyes D et al. This is an open access article distributed under the terms of the Creative Commons Attribution License, which permits unrestricted use, distribution, and reproduction in any medium, provided the original work is properly cited.

How to cite this article: Boyes D, Holland PWH, University of Oxford and Wytham Woods Genome Acquisition Lab et al. The genome sequence of the bramble shoot moth, Notocelia uddmanniana (Linnaeus, 1758) [version 1; peer review: awaiting peer review]

Wellcome Open Research 2021, 6:348 https://doi.org/10.12688/wellcomeopenres.17488.1

First published: 15 Dec 2021, 6:348 https://doi.org/10.12688/wellcomeopenres.17488.1 


\section{Species taxonomy}

Eukaryota; Metazoa; Ecdysozoa; Arthropoda; Hexapoda; Insecta; Pterygota; Neoptera; Endopterygota; Lepidoptera; Glossata; Ditrysia; Tortricoidea; Tortricidae; Olethreutinae; Eucosmini; Notocelia; Notocelia uddmanniana (Linnaeus, 1758) (NCBI: txid1594315).

\section{Background}

Notocelia uddmanniana (bramble shoot moth) is widely distributed across Western Europe and North Africa, with records further east from Kazakhstan to China. The larvae feed on brambles (Rubus sp.), occurring commonly where these species exist, and occasionally cause damage to cultivated varieties (Gordon et al., 1997). Eggs are laid singly on the foodplant, where larvae feed within a folded leaf and later within the tips of growing shoots; larvae overwinter in a silken web on the foodplant stem before recommencing feeding in spring (Dicker, 1939). Notocelia uddmanniana also occupies woodland, and is distributed widely throughout the UK, occurring more commonly in the south. The genome of $N$. uddmanniana was sequenced as part of the Darwin Tree of Life Project, a collaborative effort to sequence all of the named eukaryotic species in the Atlantic Archipelago of Britain and Ireland. Here we present a chromosomally complete genome sequence for N. uddmanniana, based on one male specimen from Wytham Woods, Oxfordshire, UK.

\section{Genome sequence report}

The genome was sequenced from a single male $N$. uddmanniana (Figure 1) collected from Wytham Woods, Oxfordshire, UK (latitude 51.772, longitude -1.338). A total of 18 -fold coverage in Pacific Biosciences single-molecule long reads (N50 $16 \mathrm{~kb}$ ) and 49-fold coverage in 10X Genomics read clouds were generated. Primary assembly contigs were scaffolded with chromosome conformation Hi-C data. Manual assembly curation corrected 165 missing/misjoins and removed 72 haplotypic duplications, reducing the assembly length by $2.16 \%$ and the scaffold number by $64.71 \%$, and increasing the scaffold N50 by $9.53 \%$.

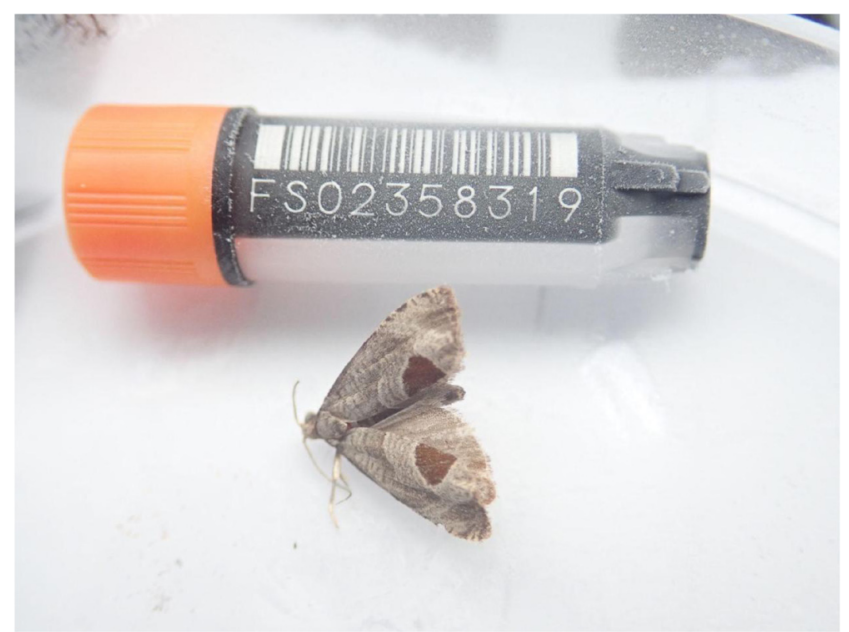

Figure 1. Image of the ilNotUddm1 specimen taken during preservation and processing.
The final assembly has a total length of $794 \mathrm{Mb}$ in 49 sequence scaffolds with a scaffold N50 of $29 \mathrm{Mb}$ (Table 1). Of the assembly sequence, $99.96 \%$ was assigned to 28 chromosomal-level scaffolds, representing 27 autosomes (numbered by sequence length), and the $\mathrm{Z}$ sex chromosome (Figure 2-Figure 5; Table 2). The assembly has a BUSCO (Simão et al., 2015) completeness of $98.9 \%$ using the lepidoptera_odb10 reference set. While not fully phased, the assembly deposited is of one haplotype. Contigs corresponding to the second haplotype have also been deposited.

\section{Methods}

Sample acquisition, DNA extraction and sequencing

A single male $M$. uddmanniana (ilNotUddm1) was collected from Wytham Woods, Oxfordshire, UK (latitude 51.772, longitude -1.338) by Douglas Boyes, UKCEH, using a light

Table 1. Genome data for Notocelia uddmanniana, ilNotUddm1.1.

\begin{tabular}{|c|c|}
\hline \multicolumn{2}{|l|}{ Project accession data } \\
\hline Assembly identifier & ilNotUddm1 \\
\hline Species & Notocelia uddmanniana \\
\hline Specimen & ilNotUddm1 \\
\hline NCBI taxonomy ID & NCBI:txid1594315 \\
\hline BioProject & PRJEB42137 \\
\hline BioSample ID & SAMEA7519916 \\
\hline Isolate information & Male, whole organism \\
\hline \multicolumn{2}{|l|}{ Raw data accessions } \\
\hline PacificBiosciences SEQUEL II & ERR6590584 \\
\hline 10X Genomics Illumina & ERR6002710-ERR6002713 \\
\hline Hi-C Illumina & ERR6002707-ERR6002709 \\
\hline \multicolumn{2}{|l|}{ Genome assembly } \\
\hline Assembly accession & GCA_905163555.1 \\
\hline Accession of alternate haplotype & GCA_905163575.1 \\
\hline Span (Mb) & 794 \\
\hline Number of contigs & 238 \\
\hline Contig N50 length (Mb) & 7 \\
\hline Number of scaffolds & 49 \\
\hline Scaffold N50 length (Mb) & 29 \\
\hline Longest scaffold (Mb) & 51 \\
\hline BUSCO* genome score & $\begin{array}{l}\text { C:98.3\%[S:97.6\%,D:0.7\%] } \\
\text { F:0.5\%,M:1.2\%,n:5286 }\end{array}$ \\
\hline
\end{tabular}

*BUSCO scores based on the lepidoptera odb10 BUSCO set using v5.1.2. $C=$ complete $[S=$ single copy, $D=$ duplicated], $F=$ fragmented, $\mathrm{M}=$ missing, $\mathrm{n}=$ number of orthologues in comparison. A full set of BUSCO scores is available at https://blobtoolkit.genomehubs.org/view/ ilNotUddm1.1/dataset/CAJHZS01/busco. 


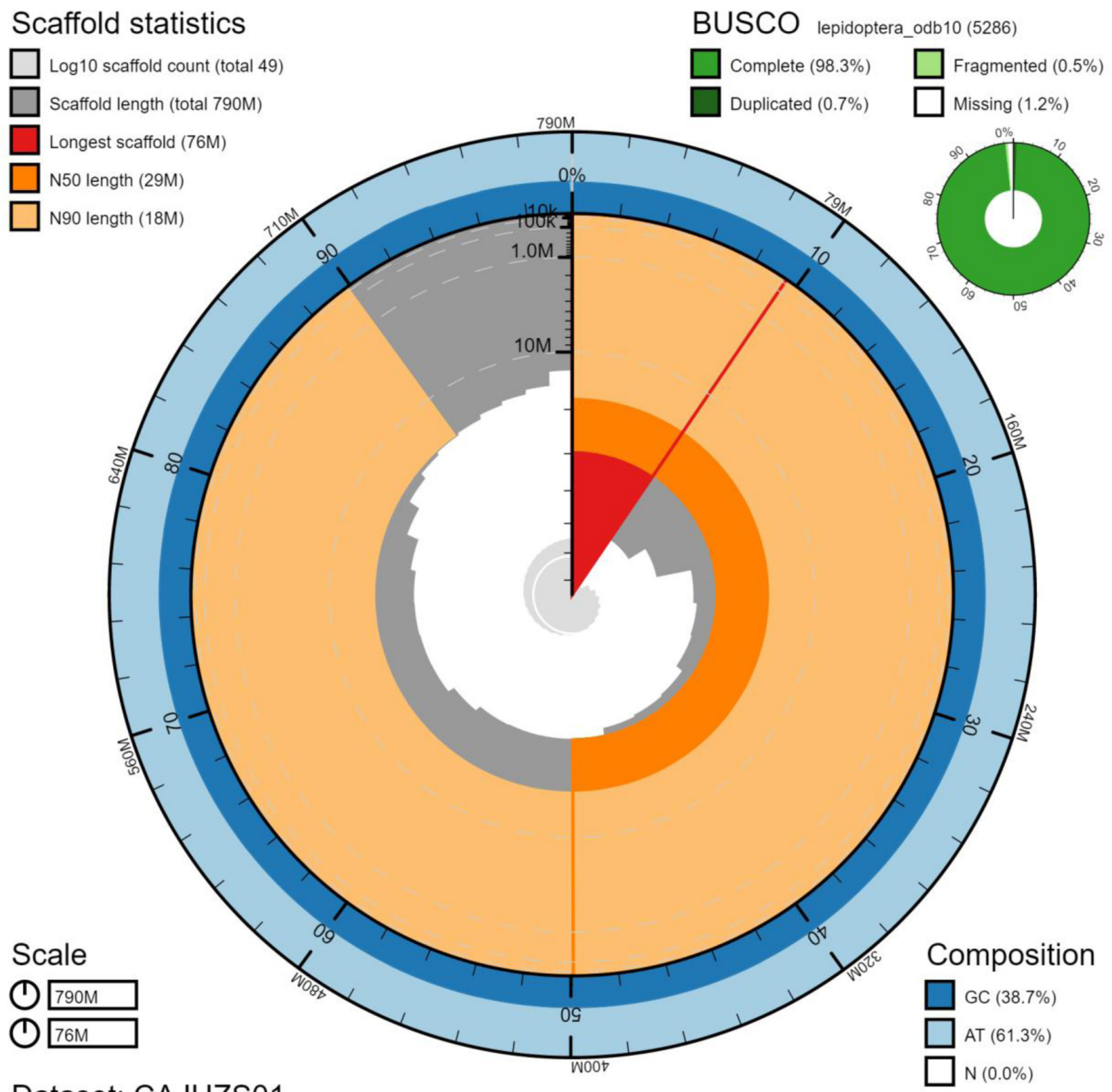

\section{Dataset: CAJHZS01}

Figure 2. Genome assembly of Notocelia uddmanniana, ilNotUddm1.1: metrics. The BlobToolKit Snailplot shows N50 metrics and BUSCO gene completeness. The main plot is divided into 1,000 size-ordered bins around the circumference with each bin representing $0.1 \%$ of the 794,123,667 bp assembly. The distribution of chromosome lengths is shown in dark grey with the plot radius scaled to the longest chromosome present in the assembly (75,621,453 bp, shown in red). Orange and pale-orange arcs show the N50 and N90 chromosome lengths (28,990,537 and 17,668,102 bp), respectively. The pale grey spiral shows the cumulative chromosome count on a log scale with white scale lines showing successive orders of magnitude. The blue and pale-blue area around the outside of the plot shows the distribution of GC, AT and N percentages in the same bins as the inner plot. A summary of complete, fragmented, duplicated and missing BUSCO genes in the lepidoptera_odb10 set is shown in the top right. An interactive version of this figure is available at https://blobtoolkit.genomehubs. org/view/ilNotUddm1.1/dataset/CAJHZS01/snail.

trap. The specimen was identified by the same individual and preserved on dry ice.

DNA was extracted from whole organism tissue at the Wellcome Sanger Institute (WSI) Scientific Operations core from the whole organism using the Qiagen MagAttract HMW DNA kit, according to the manufacturer's instructions. Pacific Biosciences HiFi circular consensus and 10X Genomics read cloud sequencing libraries were constructed according to the manufacturers' instructions. Sequencing was performed by the 


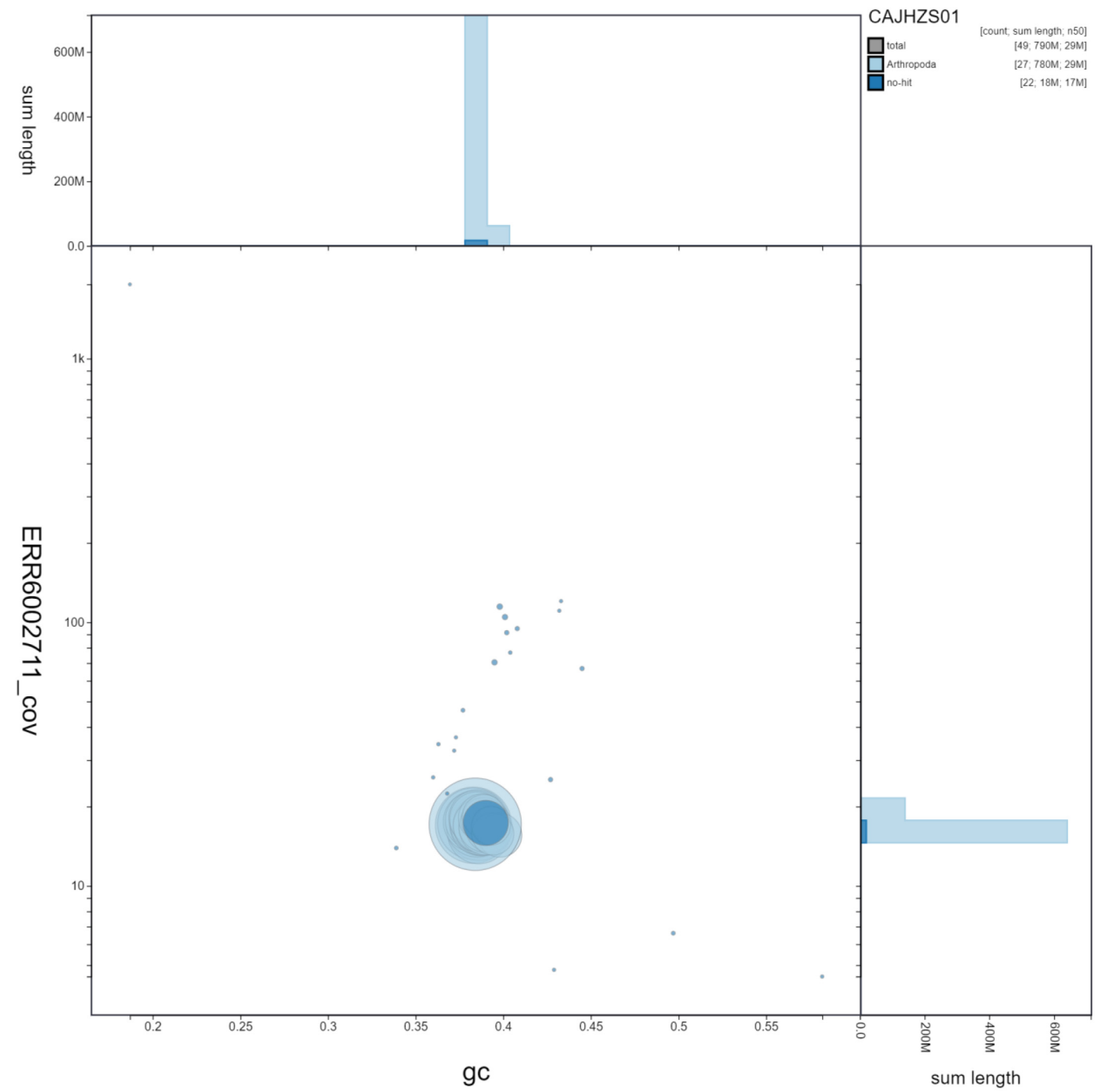

Figure 3. Genome assembly of Notocelia uddmanniana, ilNotUddm1.1: GC coverage. BlobToolKit GC-coverage plot. Scaffolds are coloured by phylum. Circles are sized in proportion to scaffold length. Histograms show the distribution of scaffold length sum along each axis. An interactive version of this figure is available at https://blobtoolkit.genomehubs.org/view/ilNotUddm1.1/dataset/CAJHZS01/blob.

Scientific Operations core at the Wellcome Sanger Institute on Pacific Biosciences SEQUEL II and Illumina HiSeq X instruments. Hi-C data were generated from remaining whole organism tissue using the Arima v1.0 kit and sequenced on HiSeq X.

\section{Genome assembly}

Assembly was carried out with Hifiasm (Cheng et al., 2021); haplotypic duplication was identified and removed with purge_dups (Guan et al., 2020), without the -e flag. One round of polishing was performed by aligning $10 \mathrm{X}$ Genomics read data to the assembly with longranger align, calling variants with freebayes (Garrison \& Marth, 2012). The assembly was then scaffolded with Hi-C data (Rao et al., 2014) using SALSA2 (Ghurye et al., 2019). The assembly was checked for contamination and corrected using the gEVAL system (Chow et al., 2016) as described previously (Howe et al., 2021). Manual curation 


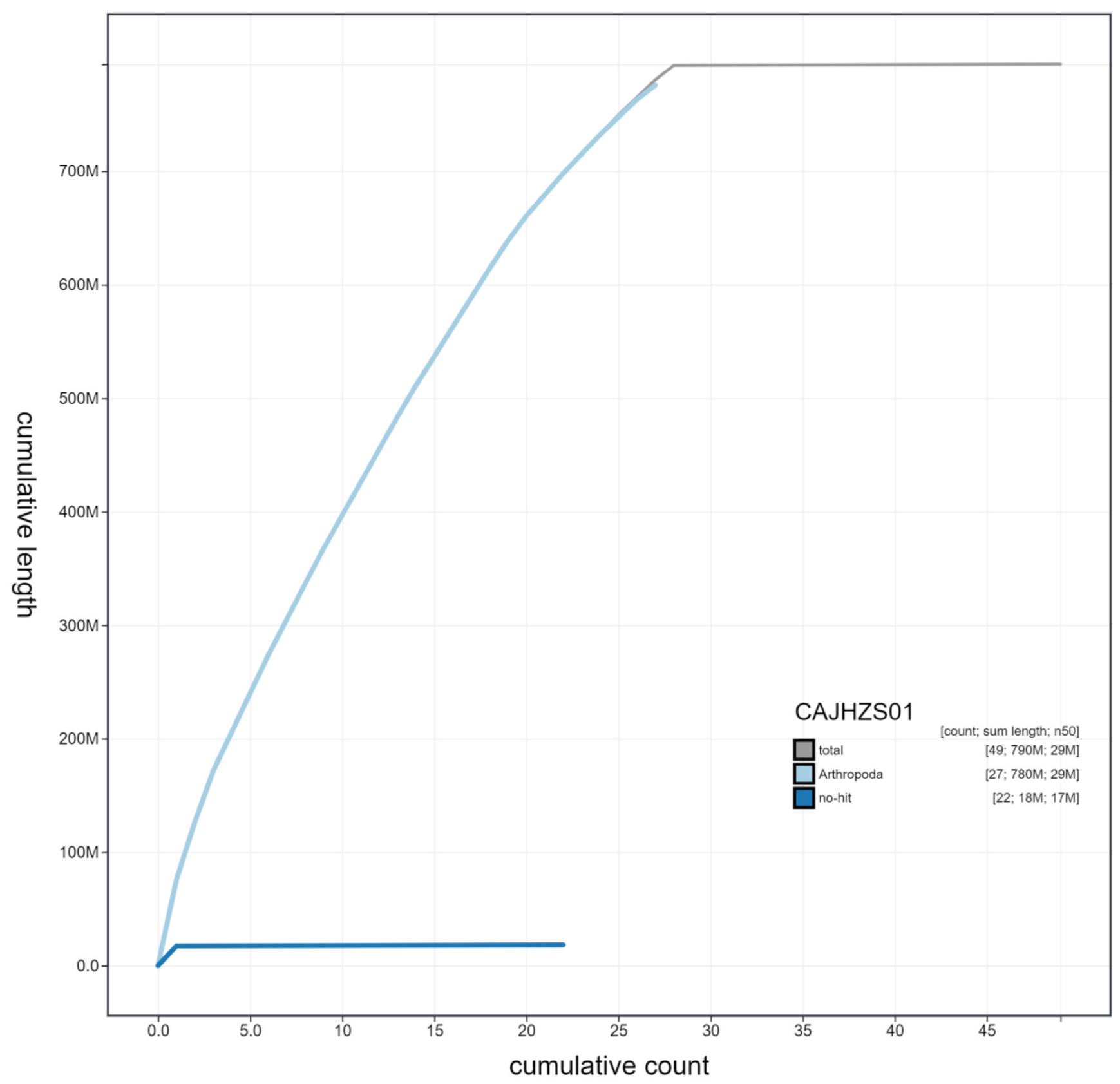

Figure 4. Genome assembly of Notocelia uddmanniana, ilNotUddm1.1: cumulative sequence. BlobToolKit cumulative sequence plot. The grey line shows cumulative length for all scaffolds. Coloured lines show cumulative lengths of scaffolds assigned to each phylum using the buscogenes taxrule. An interactive version of this figure is available at https://blobtoolkit.genomehubs.org/view/ilNotUddm1.1/dataset/ CAJHZS01/cumulative.

(Howe et al., 2021) was performed using gEVAL, HiGlass (Kerpedjiev et al., 2018) and Pretext. The mitochondrial genome was assembled using MitoHiFi (Uliano-Silva et al., 2021) and annotated using MitoFinder (Allio et al., 2020). The genome was analysed and BUSCO scores generated within the BlobToolKit environment (Challis et al., 2020). Table 3 contains a list of all software tool versions used, where appropriate.

\section{Ethics/compliance issues}

The materials that have contributed to this genome note have been supplied by a Darwin Tree of Life Partner. The submission of materials by a Darwin Tree of Life Partner is subject to the Darwin Tree of Life Project Sampling Code of Practice. By agreeing with and signing up to the Sampling Code of Practice, the Darwin Tree of Life Partner agrees they will meet the 


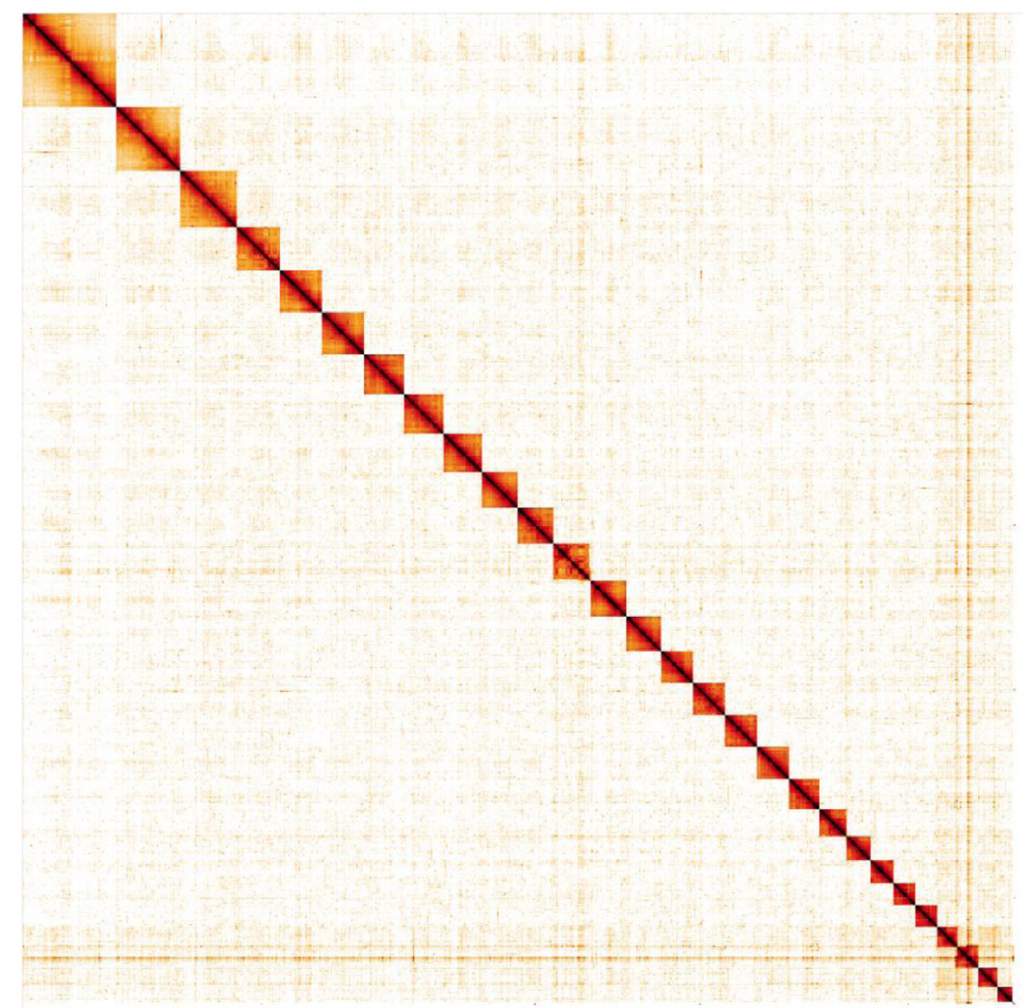

Figure 5. Genome assembly of Notocelia uddmanniana, ilNotUddm1.1: Hi-C contact map. Hi-C contact map of the ilNotUddm1.1 assembly, visualised in HiGlass. Chromosomes are given in order of size from left to right and top to bottom.

\begin{tabular}{|c|c|c|c|}
\hline $\begin{array}{l}\text { INSDC } \\
\text { accession }\end{array}$ & Chromosome & Size (Mb) & GC\% \\
\hline LR991053.1 & 1 & 51.12 & 38.3 \\
\hline LR991054.1 & 2 & 44.98 & 38.3 \\
\hline LR991055.1 & 3 & 34.90 & 38.6 \\
\hline LR991056.1 & 4 & 33.91 & 38.6 \\
\hline LR991057.1 & 5 & 33.55 & 38.5 \\
\hline LR991058.1 & 6 & 31.63 & 38.3 \\
\hline LR991059.1 & 7 & 31.24 & 38.4 \\
\hline LR991060.1 & 8 & 30.81 & 38.7 \\
\hline LR991061.1 & 9 & 29.12 & 38.8 \\
\hline LR991062.1 & 10 & 28.99 & 38.6 \\
\hline LR991063.1 & 11 & 28.96 & 38.8 \\
\hline LR991064.1 & 12 & 28.81 & 38.6 \\
\hline LR991065.1 & 13 & 27.46 & 38.5 \\
\hline LR991066.1 & 14 & 25.91 & 38.7 \\
\hline
\end{tabular}

\begin{tabular}{|c|c|c|c|}
\hline $\begin{array}{c}\text { INSDC } \\
\text { accession }\end{array}$ & Chromosome & Size (Mb) & GC\% \\
\hline LR991067.1 & 15 & 25.88 & 38.7 \\
\hline LR991068.1 & 16 & 25.71 & 38.5 \\
\hline LR991069.1 & 17 & 25.63 & 38.7 \\
\hline LR991070.1 & 18 & 24.47 & 39 \\
\hline LR991071.1 & 19 & 21.77 & 38.9 \\
\hline LR991072.1 & 20 & 19.36 & 38.8 \\
\hline LR991073.1 & 21 & 18.18 & 39.2 \\
\hline LR991074.1 & 22 & 17.67 & 38.9 \\
\hline LR991075.1 & 23 & 17.30 & 39 \\
\hline LR991077.1 & 24 & 15.87 & 38.9 \\
\hline LR991076.1 & 25 & 16.51 & 39.8 \\
\hline LR991078.1 & 26 & 15.07 & 39.4 \\
\hline LR991079.1 & 27 & 12.65 & 39.3 \\
\hline LR991052.1 & Z & 75.62 & 38.4 \\
\hline LR991080.1 & MT & 0.02 & 18.8 \\
\hline
\end{tabular}


Table 3. Software tools used.

\begin{tabular}{|l|l|l|}
\hline Software tool & Version & Source \\
\hline Hifiasm & 0.12 & Cheng et al., 2021 \\
\hline purge_dups & 1.2 .3 & Guan et al., 2020 \\
\hline SALSA2 & 2.2 & Ghurye et al., 2019 \\
\hline longranger align & 2.2 .2 & $\begin{array}{l}\text { https://support.10xgenomics.com/genome-exome/ } \\
\text { software/pipelines/latest/advanced/other-pipelines }\end{array}$ \\
\hline freebayes & $1.3 .1-17$-gaa2ace8 & Garrison \& Marth, 2012 \\
\hline MitoHiFi & 1 & https://github.com/marcelauliano/MitoHiFi \\
\hline gEVAL & N/A & Chow et al., 2016 \\
\hline HiGlass & 1.11 .6 & Kerpedjiev et al., 2018 \\
\hline PretextView & $0.1 . x$ & https://github.com/wtsi-hpag/PretextView \\
\hline BlobToolKit & 2.6 .2 & Challis et al., 2020 \\
\hline
\end{tabular}

legal and ethical requirements and standards set out within this document in respect of all samples acquired for, and supplied to, the Darwin Tree of Life Project. Each transfer of samples is further undertaken according to a Research Collaboration Agreement or Material Transfer Agreement entered into by the Darwin Tree of Life Partner, Genome Research Limited (operating as the Wellcome Sanger Institute), and in some circumstances other Darwin Tree of Life collaborators.

\section{Data availability}

European Nucleotide Archive: Notocelia uddmanniana (bramble shoot). Accession number PRJEB42137: https://www.ebi.ac.uk/ ena/browser/view/PRJEB42037.

The genome sequence is released openly for reuse. The $N$. uddmanniana genome sequencing initiative is part of the Darwin Tree of Life (DToL) project. All raw sequence data and the assembly have been deposited in INSDC databases. The genome will be annotated and presented through the Ensembl pipeline at the European Bioinformatics Institute. Raw data and assembly accession identifiers are reported in Table 1.

\section{Author information}

Members of the University of Oxford and Wytham Woods Genome Acquisition Lab are listed here: https://doi.org/10.5281/ zenodo.5746938.

Members of the Darwin Tree of Life Barcoding collective are listed here: https://doi.org/10.5281/zenodo.5744972.

Members of the Wellcome Sanger Institute Tree of Life programme are listed here: https://doi.org/10.5281/zenodo. 5744840

Members of Wellcome Sanger Institute Scientific Operations: DNA Pipelines collective are listed here: https://doi.org/10.5281/ zenodo. 5746904 .

Members of the Tree of Life Core Informatics collective are listed here: https://doi.org/10.5281/zenodo.5743293.

Members of the Darwin Tree of Life Consortium are listed here: https://doi.org/10.5281/zenodo.5638618.
Allio R, Schomaker-Bastos A, Romiguier J, et al.: MitoFinder: Efficient automated large-scale extraction of mitogenomic data in target enrichment phylogenomics. Mol Ecol Resour. 2020; 20(4): 892-905. PubMed Abstract | Publisher Full Text | Free Full Text

Challis R, Richards E, Rajan J, et al.: BlobToolKit - Interactive Quality Assessment of Genome Assemblies. G3 (Bethesda). 2020; 10(4): 1361-74. PubMed Abstract | Publisher Full Text | Free Full Text

Cheng $\mathrm{H}$, Concepcion GT, Feng X, et al.: Haplotype-Resolved de Novo Assembly Using Phased Assembly Graphs with Hifiasm. Nat Methods. 2021; 18(2): 170-75.

PubMed Abstract | Publisher Full Text | Free Full Text
Chow W, Brugger K, Caccamo M, et al.: gEVAL - a Web-Based Browser for Evaluating Genome Assemblies. Bioinformatics. 2016; 32(16): 2508-10. PubMed Abstract | Publisher Full Text | Free Full Text

Dicker GHL: The Morphology and Biology of the Bramble Shoot-Webber, Notocelia Uddmanniana L. (Tortricidae). Ann Appl Biol. 1939; 26(4): 710-38. Publisher Full Text

Garrison E, Marth G: Haplotype-Based Variant Detection from Short-Read Sequencing. arXiv: 1207.3907. 2012.

Reference Source

Ghurye J, Rhie A, Walenz BP, et al.: Integrating Hi-C Links with Assembly Graphs 
for Chromosome-Scale Assembly. PLoS Comput Biol. 2019; 15(8): e1007273. PubMed Abstract | Publisher Full Text | Free Full Text

Gordon SC, Woodford JAT, Birch ANE: Arthropod Pests of Rubus in Europe: Pest Status, Current and Future Control Strategies. J Hortic Sci. 1997; 72(6): 831-62.

Publisher Full Text

Guan D, McCarthy SA, Wood J, et al:: Identifying and Removing Haplotypic Duplication in Primary Genome Assemblies. Bioinformatics. 2020; 36(9): 2896-98.

PubMed Abstract | Publisher Full Text | Free Full Text

Howe K, Chow W, Collins J, et al.: Significantly Improving the Quality of Genome Assemblies through Curation. GigaScience. 2021; 10(1): giaa153. PubMed Abstract | Publisher Full Text | Free Full Text

Kerpedjiev P, Abdennur N, Lekschas F, et al.: HiGlass: Web-Based Visual
Exploration and Analysis of Genome Interaction Maps. Genome Biol. 2018; 19(1): 125.

PubMed Abstract | Publisher Full Text | Free Full Text

Rao SS, Huntley MH, Durand NC, et al:: A 3D Map of the Human Genome at Kilobase Resolution Reveals Principles of Chromatin Looping. Cell. 2014; 159(7): 1665-80.

PubMed Abstract | Publisher Full Text | Free Full Text

Simão FA, Waterhouse RM, Ioannidis P, et al.: BUSCO: Assessing Genome Assembly and Annotation Completeness with Single-Copy Orthologs. Bioinformatics. 2015; 31(19): 3210-12.

PubMed Abstract | Publisher Full Text

Uliano-Silva M, Nunes JGF, Krasheninnikova K, et al.: marcelauliano/MitoHiFi: mitohifi_v2.0. 2021.

Publisher Full Text 